\title{
Le Swiss Quality Award tient toutes ses promesses
}

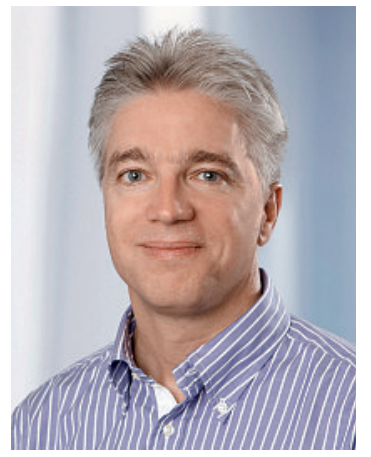

Il y a des prix que l'on ne souhaite pas forcément gagner, à l'instar d'un prix bien connu visant à promouvoir l'économie et dont la moitié des lauréats ont fait faillite quelques années après l'avoir remporté. Mais le Swiss Quality Award (www.swissqualityaward.ch) est différent, nous en sommes persuadés, d'ailleurs les réactions - toutes optimistes - des lauréats de l'année dernière le montrent bien: «notre projet a tout à coup été pris au sérieux au sein de la clinique... notre travail a été reconnu... et cela nous a également permis de lier de précieux contacts en dehors de la clinique...», ou «le projet nous a amené beaucoup de publicité...; et le prix nous a permis de financer du matériel afin de poursuivre notre étude clinique pilote...»

Et ce sont précisément ces objectifs qui sont à l'origine du Swiss Quality Award: accroître la visibilité des projets,

\section{Les réactions des lauréats de l'année dernière sont toutes optimistes.}

aussi bien à l'intérieur qu'à l'extérieur des institutions, permettre aux spécialistes de se rencontrer et de développer leur réseau, et leur apporter un soutien direct afin qu'ils puissent poursuivre leurs travaux. Avec le Symposium national pour la gestion de la qualité dans le secteur de la santé, la remise du Swiss Quality Award entend servir de plateforme d'échange pour les spécialistes, tout en donnant l'occasion à ces derniers de présenter leurs projets qualité, achevés ou en cours d'élaboration, à un public plus large. Et en ce sens, non seulement les lauréats mais également tous les participants ont à y gagner.
Suite au succès de l'édition 2011 - lors de laquelle plus de 100 projets ont été soumis - nous espérons que le Swiss Quality Award 2012 poursuivra sur cette excellente lancée. Les trois organisateurs, la FMH, la SQMH et l'IEFM, travaillent déjà d'arrache-pied en prévision de ce concours qui, hormis quelques légères modifications organisationnelles, se déroulera comme à l'accoutumée, la méthode ayant porté ses fruits. Afin de continuer à englober une palette de projets aussi large que possible, le nombre de catégories reste inchangé: vous retrouverez les quatre grands thèmes Manage-

\section{Nous ne recherchons pas uniquement des travaux issus du monde acadé- mique: les idées inspirées de la pra- tique sont aussi les bienvenues.}

ment, Sécurité des patients, Technologie et Empowerment. Nous tenons une fois encore à souligner que nous ne recherchons pas uniquement des travaux issus du monde académique, mais que les idées inspirées de la pratique sont aussi les bienvenues. Il est également possible de présenter à nouveau un projet déjà soumis en 2011, mais non primé. Beaucoup de choses peuvent changer en une année: la période d'observation est plus longue, le nombre de patients a augmenté ou de nouveaux résultats ont peut-être été trouvés autant d'éléments qui permettent de perfectionner le projet. Il peut donc être intéressant de mettre à jour des travaux en cours.

Nous nous réjouissons d'ores et déjà de découvrir vos idées novatrices et prometteuses et de vous rencontrer le 15 mai 2012 lors de la remise des prix à Berne!

Dr Daniel Herren MHA, membre du Comité central de la FMH, responsable du domaine DDQ 